\title{
10 Fazit
}

Ziel dieser Arbeit war, Vertrauen in journalistische Medien vor dem Hintergrund des digitalen Strukturwandels von Medien und Öffentlichkeit zu untersuchen. Im Mittelpunkt stand dabei mit dem generalisierten Vertrauen in Journalismus eine allgemeine Attitüde gegenüber dem Journalismus als solchem. Die Forschungsfragen wurden in vier Fragenblöcken systematisiert: 1) Grundlagen und Verständnis von Vertrauen in Journalismus aus Sicht der Rezipient_innen sowie die Zusammenhänge von Vertrauen in Journalismus mit 2) allgemeinen Personenmerkmalen, 3) Diskrepanzen aus Erwartungen an Journalismus und der Wahrnehmung seiner Berichterstattung sowie 4) der Informationsnutzung, wobei hier insbesondere der Kontakt mit alternativen Ereignisdarstellungen und Medienkritik im Internet im Mittelpunkt stand. Vertrauen in Journalismus wurde dabei als langfristiges Konstrukt bestimmt, das die Bereitschaft bezeichnet, Aussagen journalistischer Medien in das eigene Meinungs-, Einstellungs- und Verhaltensspektrum zu übernehmen. Die Forschungsfragen wurden auf Basis einer Methodenkombination aus qualitativen Leitfadeninterviews mit $n=13$ Bürger_innen sowie einer repräsentativen Online-Befragung unter $n=936$ Personen beantwortet.

\subsection{Zusammenfassung der Ergebnisse}

Die Ergebnisse zeigen zunächst, dass Rezipient_innen ein relativ klar abgegrenztes Bild etablierter, journalistischer Mainstream-Medien im Kopf haben, das vor allem große Tageszeitungen, Magazine und den öffentlich-rechtlichen Rundfunk sowie ihre Online-Ableger umfasst. Sie bringen diesem abstrakten Bezugsobjekt ein generalisiertes Vertrauen entgegen und sind in der Lage, Erwartungen an den Journalismus als solchen ebenso zu formulieren wie grundsätzliche Einschätzungen zu seiner Qualität zu geben. Wenn auch einzelnen Quellen mehr oder weniger Vertrauen entgegengebracht wird, so zeigt sich doch eine generelle Vertrauens- bzw. Misstrauensneigung gegenüber journalistischen Medien.

Diese journalistischen Medien genießen in Deutschland aktuell durchaus großen Rückhalt in der Bevölkerung: Die deskriptiven Vertrauenswerte liegen etwa im mittleren Bereich mit einem leichten Überhang zu hohem Vertrauen. Allerdings 
zeigt sich eine substanziell große Gruppe von 10-15 Prozent der Befragten, die extrem niedriges Vertrauen in Journalismus angeben. Das Misstrauen in Journalismus wird vor allem von Personenmerkmalen geprägt. Typische Medienskeptiker sind vor allem solche Personen, die auch ihren Mitmenschen misstrauen, die mit dem politischen System unzufrieden sind und an mächtige Verschwörungen im Hintergrund glauben. Medienskeptiker haben darüber hinaus ein größeres Bedürfnis nach einer klaren Einteilung in Richtig und Falsch. Sie finden sich vor allem am rechten Rand des politischen Spektrums und unter Nichtwählern, so dass insbesondere das rechtspopulistische und politisch entfremdete Milieu wenig Vertrauen in journalistische Medien hat. Gleichzeitig muss beachtet werden, dass es sich dabei um Durchschnittswerte handelt: Prinzipiell finden sich Personen mit niedrigem Vertrauen in so gut wie allen gesellschaftlichen Milieus.

In Übereinstimmung sowohl der Leitfadeninterviews als auch der quantitativen Untersuchung konnte festgestellt werden, dass das Vertrauen hinsichtlich der Qualitätswahrnehmungen vor allem von der Wahrnehmung von Richtigkeit, der vollständigen Auswahl relevanter Themen, der Trennung von Nachricht und Meinung sowie von Seriosität bzw. Sachlichkeit geprägt ist. Die quantitative Untersuchung konnte zusätzlich zeigen, dass Vertrauen in Journalismus unter den Personen höher ist, die ihre Meinung in den Medien widergespiegelt sehen (auch unter Kontrolle aller relevanter Personenmerkmale). Bedeutsam für das Vertrauen sind jenseits der Wahrnehmung der Berichterstattung aber vor allem die wahrgenommenen Gründe für Qualitätsmängel. Der stärkste Treiber für Misstrauen ist in diesem Bereich die Wahrnehmung, dass Fehler im Journalismus das Resultat absichtlicher Manipulationen sind - unabhängig davon, ob die Befragten diese Vorwürfe begründen können oder nicht. Die Wahrnehmung unabsichtlicher Fehler ist hingegen mit höherem Vertrauen verknüpft. Personen, die Fehler als menschlich und unabsichtlich wahrnehmen, verzeihen wahrgenommene Qualitätsmängel der Berichterstattung außerdem eher: Bei ihnen sind die Wahrnehmungen der Qualität der Berichterstattung weniger ausschlaggebend für Vertrauen. Diese Zusammenhänge haben sich so vor allem bei der intuitiven Operationalisierung von Vertrauen in Journalismus gezeigt. Fragt man hingegen danach, inwiefern Rezipient_innen Risiken in Bezug auf Journalismus eingehen, fallen einige Einflussfaktoren der Qualitätswahrnehmungen weg und die Wahrnehmung der Widerspiegelung der eigenen Meinung hat einen stärkeren Einfluss: Ob sich Menschen bei ihrer Meinungsbildung, in der Anschlusskommunikation und bei wichtigen Entscheidungen also auf die Informationen journalistischer Medien verlassen, hängt vor allem davon ab, ob die Menschen ihre Meinungen in diesen Medien repräsentiert sehen. Die wahrgenommene Richtigkeit der Inhalte hat darauf hingegen keinen Einfluss.

Hinsichtlich der Informationsnutzung zeigt sich, dass zwar journalistische Medien nach wie vor die Informationsrepertoires deutscher Onliner dominieren, 
nicht-journalistische Quellen aber einen festen Platz darin einnehmen und durchaus auf substanzielle Nutzungszahlen verweisen können. Hinsichtlich der Informationskanäle ist vor allem der Zugriff über algorithmisch personalisierte Kanäle relevant (z. B. soziale Netzwerkseiten, Suchmaschinen, Videoplattformen), die für die Befragten die wichtigsten Wege zu Nachrichten sind. Die Zusammenhänge von Informationsnutzung mit dem Vertrauen in Journalismus zeigen, dass die Informationsnutzung relativ wenig Varianz im Vertrauen erklären kann. Vertrauen in journalistische Medien hängt also offenbar deutlich stärker an Personenmerkmalen und Qualitätswahrnehmungen und korrespondiert nur schwach mit der Nachrichtennutzung. Dabei wenden sich Medienskeptiker keineswegs von aktuellen Informationen oder von journalistischen Medien ab, im Gegenteil liegt die absolute Informationsnutzung über alle Quellen bei Personen mit niedrigem Vertrauen sogar höher als bei solchen mit hohem Vertrauen. Unter der Kontrolle von Personenmerkmalen zeigt sich, dass die Nutzung journalistischer Medien außerdem nicht positiv mit dem Vertrauen korreliert. Medienskeptiker nutzen allerdings intensiver Alternativmedien. Hingegen hängt die Nutzung von Originalquellen und Nutzerkommentaren nur dann negativ mit dem Vertrauen in Journalismus zusammen, wenn darin alternative Ereignisdarstellungen und Medienkritik rezipiert werden. Der Kontakt mit diesen Inhalten korrespondiert wiederum nur bei diesen Personen negativ mit Vertrauen in Journalismus, die absichtlich danach suchen. Damit ein negativer Effekt alternativer Ereignisdarstellungen und Medienkritik auf Vertrauen in Journalismus eintreten kann, braucht es also eine aktive Suche nach diesen Inhalten, ein Vertrauensverlust, en passant' ist kaum zu erwarten. Die qualitative Untersuchung hat allerdings gezeigt, dass zufälliger Kontakt mit diesen Inhalten durchaus als Initialzündung für eine Abwärtsspirale aus Misstrauen und Rezeption alternativer Darstellungen auftreten kann. Die Rezeption alternativer Ereignisdarstellungen im Internet kann also als ein Katalysator zur Verstärkung medienskeptischer Einstellungen gelten.

Schließlich ließen sich sowohl unter den Personen mit hohem als auch mit niedrigem Vertrauen drei Gruppen identifizieren, die sich hinsichtlich ihres Medienwissens unterscheiden. Bei den Medienskeptikern sind die ,enttäuschten Journalismus-Idealisten“ eine Gruppe hoch gebildeter Personen, die über Journalismus gut Bescheid wissen, sich stark für Nachrichten interessieren und auch hohe Erwartungen an Journalismus haben. Sie verwenden intensiv nicht-journalistische Quellen und suchen auch aktiv nach alternativen Ereignisdarstellungen und Medienkritik. Demgegenüber steht eine Gruppe ,abgehängter Skeptiker' mit sehr niedrigem Wissen, geringen Erwartungen und äußerst geringer Informationsnutzung sowie die ,diffus Medienskeptischen', die mittleres Wissen über Journalismus aufweisen. Analog finden sich ähnliche Gruppen auf Seiten der Personen mit hohem Vertrauen in Journalismus. Für einen Überblick fasst Abbildung 23 nochmal einige der zentralen Ergebnisse der Arbeit zusammen. 


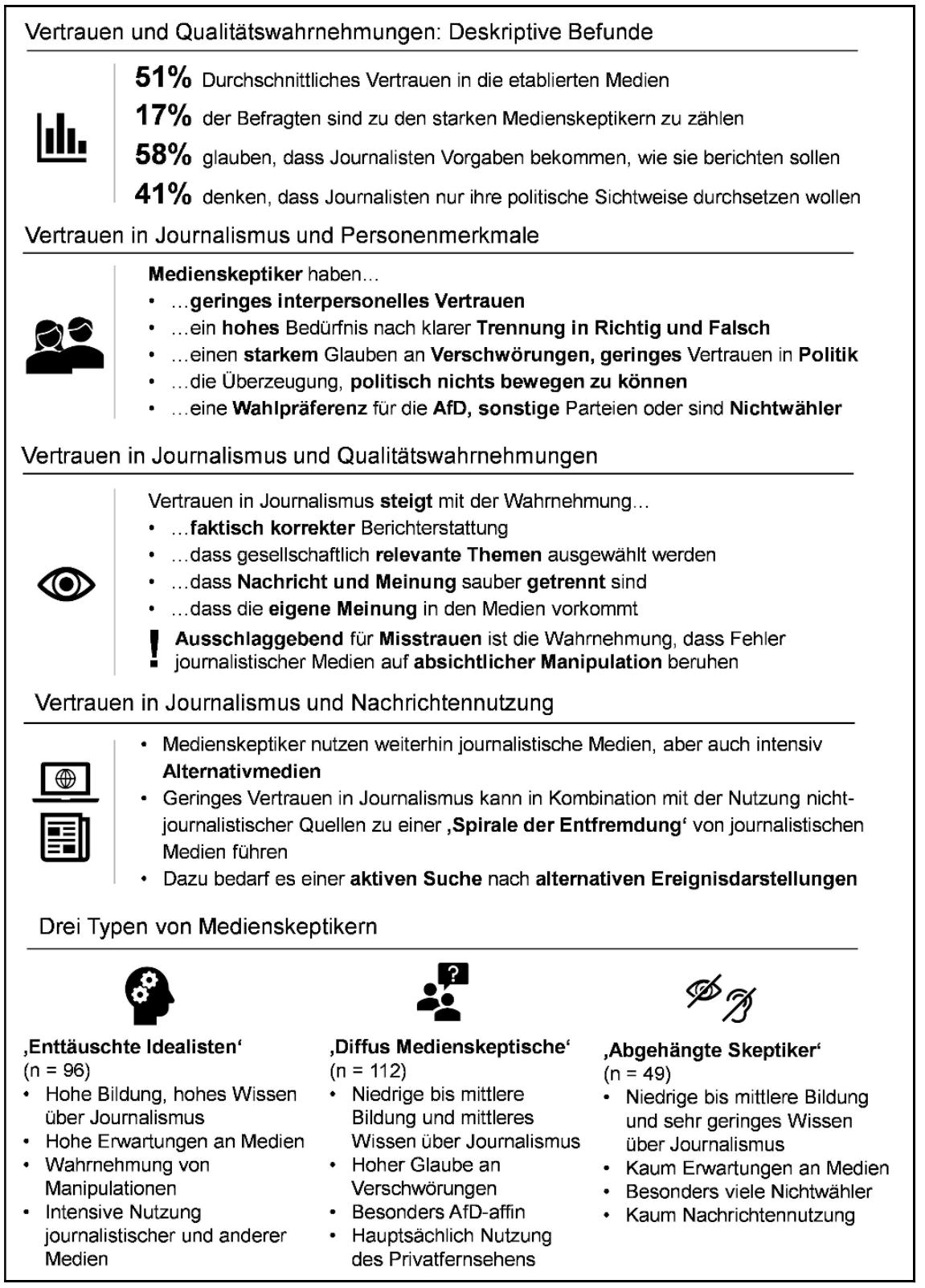

Abbildung 23: Zusammenfassung der zentralen Ergebnisse 


\subsection{Diskussion und weiterführende Forschung}

In Kapitel 5.1 wurden fünf zentrale Forschungsdefizite in der Medienvertrauensforschung identifiziert, die die vorliegende Studie adressiert hat. Das folgende Kapitel diskutiert, inwiefern diese Forschungslücken geschlossen werden konnten und geht dabei auf die Limitationen und Stärken der vorliegenden Studie ein. Dabei werden auch weitere theoretische sowie methodisch-konzeptuelle Erkenntnisse abgeleitet, die weitere Forschung anregen können.

Das erste Forschungsdefizit betraf das Verständnis von generalisiertem Vertrauen in Journalismus bei den Rezipient_innen. Die Ergebnisse der Leitfadeninterviews konnten zeigen, dass sich bei den Befragten zwar ein generalisiertes Vertrauen als Attitüde gegenüber der Gesamtheit journalistischer Medien zeigt, offensichtlich ist hier aber für zukünftige Forschung noch weitere Differenzierung nötig. So hängt Vertrauen in unterschiedliche mediale Bewertungsobjekte zwar stark zusammen, wie die Leitfadeninterviews zeigen konnten (siehe auch Kiousis, 2001, S. 396). Allerdings machen die Befragten durchaus themen- und markenspezifische Unterschiede im Vertrauen. So genießt etwa der öffentlichrechtliche Rundfunk ebenso wie Qualitätszeitungen besonders hohes Vertrauen, während die Befragten Boulevardmedien skeptischer gegenüberstehen. Es erscheint daher lohnend, diese Unterschiede bei zukünftiger Forschung in den Blick zu nehmen und vor allem die Bezüge des generalisierten Vertrauens in Journalismus mit Vertrauen in Einzelmedien genauer zu betrachten.

Darüber hinaus war vor allem in der qualitativen Studie auffällig, dass die Gruppe der abgehängten Personen mit einer Gleichgültigkeit gegenüber Medien kaum erreicht werden konnte. Dieser Befund weist auf eine theoretische ebenso wie eine empirische Leerstelle der Forschung zu Vertrauen in Journalismus hin: Das Verhältnis von Gleichgültigkeit gegenüber journalistischen Medien und Vertrauen in Journalismus ist bislang kaum genauer exploriert. Die bisherige Forschung beschäftigt sich intensiv mit Extremgruppen hohen bzw. niedrigen Vertrauens, die damit ein gewisses Involvement gegenüber Journalismus haben. Bislang weitgehend unberücksichtigt blieben Personen, für die Vertrauen in Journalismus überhaupt keine Kategorie ist bzw. die vom medialen und politischen Tagesgeschehen aus unterschiedlichen Gründen abgekoppelt sind. Die vorliegende Studie hat mit der Identifikation abgehängter Skeptiker und uninformierter Journalismus-Fans einen ersten Schritt getan, hier ist aber noch mehr - insbesondere auch qualitative - Forschung notwendig. Theoretisch und empirisch kann dabei an die Forschung zur Nachrichtenvermeidung angeknüpft werden und sie systematisch mit Vertrauen in Journalismus in Verbindung gebracht werden (Trilling \& Schoenbach, 2013).

Die mangelnde Berücksichtigung von Gleichgültigkeit gegenüber Journalismus hängt auch mit dem zweiten Forschungsdefizit zusammen, der Operatio- 
nalisierung von Vertrauen in Journalismus. Misst man Vertrauen auf einer Skala von keinem Vertrauen bis hohem Vertrauen, finden sich vermutlich im Bereich mittleren Vertrauens sowohl Personen mit einer kritischen, balancierten Haltung gegenüber dem Journalismus als auch Personen, die dem Journalismus gleichgültig gegenüberstehen. Hier müssen zukünftig Messverfahren entwickelt werden, die eine Differenzierung erlauben. In der vorliegenden Studie wurde Vertrauen in Journalismus einerseits mit einer direkten Ein-Item-Messung operationalisiert (intuitive Messung), andererseits wurde ein neuer Operationalisierungsvorschlag umgesetzt. Dabei wurde Vertrauen darüber gemessen, ob die Befragten sich bei ihrer Meinungsbildung und in der Anschlusskommunikation auf journalistische Medien verlassen (instrumentelle Messung). Die Varianzaufklärung in der intuitiven Messung lag dabei durchgehend höher als in der instrumentellen Messung, was wohl auf Konfundierungen mit der generellen Bereitschaft zu Anschlusskommunikation bzw. dem Interesse für aktuelle politische Ereignisse zurückzuführen ist. Damit konnten zwar interessante Zusammenhänge insbesondere mit Qualitätswahrnehmungen identifiziert werden; die Operationalisierung ist aber hier sicherlich verbesserungsbedürftig. Theoretisch scheint diese Variante nach wie vor vielversprechend, insbesondere für eine Konsolidierung der Forschung zu Vertrauen in Journalismus, die sich bislang eher zögerlich systematisch mit dem zugrundeliegenden Verständnis von Vertrauen auseinandergesetzt hat. Der Begriff Vertrauen wird in der Literatur überwiegend als Klammerbegriff verwendet, unter dem im weitesten Sinne Einstellungen gegenüber journalistischen Medien verhandelt werden. Wird Vertrauen allerdings als theoretisches Konstrukt ernst genommen, sollten zukünftige Operationalisierungen die Risiko- und Handlungskomponente des Vertrauens berücksichtigen. Die in dieser Studie entwickelte Skala kann dafür ein Ausgangspunkt sein.

Das dritte Forschungsdefizit betraf Leerstellen und widersprüchliche Ergebnisse beim Einfluss von Personenmerkmalen. Die Studie konnte hier einerseits eine Reihe von bekannten Zusammenhängen bestätigen, andererseits einige Lücken füllen. So wurde etwa die konstruktivistische Weltsicht als weitere relevante Persönlichkeitseigenschaft identifiziert, die beschreibt, inwiefern Personen davon ausgehen, dass es häufig Ansichtssache ist, was stimmt und was nicht. Je eher Personen hier eine klare Trennung in Richtig und Falsch brauchen, umso geringer ist ihr Vertrauen in Journalismus. Außerdem handelt es sich um eine der ersten Studien, die den Zusammenhang von politischer Selbstwirksamkeitsüberzeugung und Vertrauen in Journalismus untersucht hat und bestätigen konnte, dass insbesondere politische Einflussüberzeugung positiv mit dem Vertrauen in Journalismus korreliert. Bislang noch kaum untersucht sind außerdem die $\mathrm{Zu}$ sammenhänge von Vertrauen in Journalismus mit dem wahrgenommenen Vertrauensklima in der Bevölkerung, wo die Studie einen ersten Beitrag leisten konnte. So zeigten sich deutliche Korrelationen zwischen der wahrgenommenen 
Bevölkerungsmeinung gegenüber Journalismus mit der eigenen Meinung und es konnten auf Seiten der Medienskeptiker eine gefühlte Avantgarde sowie eine gefühlte Mehrheit identifiziert werden. Ungeklärt ist jedoch die Richtung des Zusammenhangs von eigenem Vertrauen und der Einschätzung des Vertrauensklimas. Eine offene Frage ist hier insbesondere, ob und inwieweit die Diskussion von (vermeintlichen) Vertrauensproblemen das tatsächliche Vertrauen der Bevölkerung beeinflusst. Anzunehmen ist, dass gerade im Zuge der LügenpresseDebatte der vergangenen Jahre die öffentliche Diskussion für die Polarisierungstendenzen des Vertrauens in Journalismus mit verantwortlich war (Ziegele et al., 2018, S. 160). Darüber hinaus ist zu klären, inwiefern kleine Gruppen von Medienskeptikern durch intensive öffentliche Kritik am Journalismus die Vertrauensklimawahrnehmung beeinflussen und damit einen indirekten Einfluss auf das Vertrauen ausüben.

Darüber hinaus konnte die Studie einen Beitrag zum Zusammenhang des Medienwissens mit Vertrauen leisten. Hier wurden mehrere Typen identifiziert, die sich in ihrem Wissen über journalistische Medien deutlich unterscheiden und auf dieser Basis auch unterschiedliches Vertrauen oder Misstrauen äußern. Daraus lässt sich ableiten, dass die widersprüchlichen Ergebnisse vieler Studien zu Personenmerkmalen offenbar mit nicht-linearen Zusammenhängen zu tun haben: So gibt es sowohl niedrig-, wie auch hoch gebildete Medienskeptiker, deren Misstrauen jedoch auf unterschiedlichem Wissen und anderen Erwartungen gegenüber Journalismus fußt. Diesen und weitere ähnliche Zusammenhänge kann man aber kaum entdecken, wenn ausschließlich lineare Zusammenhänge mit dem Vertrauen untersucht werden. Insgesamt wäre es daher lohnend, die rein lineare Logik vieler Untersuchungen zu hinterfragen. Die zukünftige Forschung sollte sich daher mithilfe von Moderations- und Mediationsanalysen stärker um die Fragen des Wie und Warum der Zusammenhänge von Vertrauen in Journalismus mit anderen Faktoren bemühen und nach differenzierten Zusammenhängen in unterschiedlichen Bevölkerungsgruppen suchen.

Das vierte Forschungsdefizit betraf den Zusammenhang von Qualitätswahrnehmungen und Vertrauen in Journalismus. Dabei hat sich als ein wesentlicher Mehrwert der Studie der Ansatz herauskristallisiert, Qualitätswahrnehmungen und Vertrauen getrennt voneinander zu operationalisieren und die Zusammenhänge zu betrachten, anstatt Vertrauen mit Qualitätswahrnehmungen gleichzusetzen. So konnte herausgearbeitet werden, dass die wahrgenommene Qualität der Berichterstattung nur einen Teil der Varianz im Vertrauen erklären kann und vor allem die wahrgenommenen Gründe für Qualitätsmängel ausschlaggebend sind. Für diese Studie muss jedoch kritisch angemerkt werden, dass die Abfrage der spezifischen Qualitätserwartungen und -wahrnehmungen auf der Ebene der Berichterstattung journalistischer Medien in ihrer Gesamtheit offenbar zu detailliert für die Befragten ist. Die hohen Korrelationen zwischen den Items weisen 
auf eine relativ holistische Einschätzung der Qualität hin, die sich letztlich auf ein Gut-Schlecht-Urteil zurückführen lässt. Die vertrauensrelevanten Qualitätswahrnehmungen konnten zwar auf einige Kernaspekte reduziert werden, die sich mit den Erkenntnissen der qualitativen Untersuchung decken. Dennoch ist es für die zukünftige Forschung eine zentrale Aufgabe, einen kritischen Blick auf diese Arten der Messung zu legen. Die Studie stellt dennoch eine der ersten dar, die Qualitätswahrnehmungen des Publikums systematisch mit ihren Vertrauenseinschätzungen verknüpft und exploriert, in welchem Verhältnis Qualitätszuschreibungen und Vertrauen stehen.

Hinsichtlich der Zusammenhänge von Erwartungs-Wahrnehmungs-Diskrepanzen mit dem Vertrauen in Journalismus hat sich das Diskrepanzmodell als nicht erklärungsstark erwiesen. Die Varianzaufklärung im Vertrauen lag jeweils deutlich höher, wenn allein die Qualitätswahrnehmungen eingesetzt wurden. Der Diskrepanz-Ansatz wurde daher verworfen und stattdessen die Zusammenhänge mit den Wahrnehmungen der Qualität journalistischer Berichterstattung untersucht. Auch hier kann zukünftige Forschung ansetzen, denn der DiskrepanzAnsatz scheint theoretisch nach wie vor vielversprechend. Anzunehmen ist, dass mit einer anderen Abfrage von (weniger) Qualitätsdimensionen evtl. auch eine bessere Varianzaufklärung zu leisten ist. Zu vermuten steht außerdem, dass konkretere Bezugsobjekte als journalistische Medien generell hier sinnvoller sind.

Hinsichtlich des letzten Forschungsdefizits, dem Zusammenhang von Vertrauen in Journalismus mit der Informationsnutzung, hat sich zunächst die Abfrage der Informationsnutzung über vier Online-Informationsquellen und den zugehörigen Kanälen als sinnvolle Variante erwiesen. Damit können Vermischungen von Informationsquellen und -kanälen vermieden werden und genauer untersucht werden, welche Inhalte eigentlich rezipiert werden, wenn die Nutzung sozialer Netzwerkseiten oder von Suchmaschinen abgefragt wird. Ungeachtet aller Probleme der Selbstauskunft liegt damit eine nun in zwei Studien validierte Art der Messung differenzierter Informationsnutzung im Internet vor, die plausible Ergebnisse liefert (siehe auch Schweiger et al., 2019). Durch die verwendete Abfrageform ist hier jedoch nicht zu klären, was die Befragten jeweils unter Alternativmedien oder Originalquellen verstehen. Trotz der Nennung von Beispielen in der Frage ist es also wahrscheinlich, dass die Befragten nicht in allen Fällen sauber zwischen journalistischen und nicht-journalistischen Quellen trennen (können). Die qualitative Erhebung hat jedoch gezeigt, dass insbesondere die intensiven Nutzer von Alternativmedien durchaus eine klare Vorstellung davon haben, was unter diese Medien fällt und dass sie sie klar vom journalistischen Mainstream trennen. Durch die Abfrage in Selbstauskunft konnte außerdem nur ermittelt werden, ob die Befragten wahrnehmen, inwiefern nicht-journalistische Quellen alternative Ereignisdarstellungen liefern oder den Journalismus kritisieren. Welche Inhalte tatsächlich rezipiert wurden, kann damit nicht geklärt wer- 
den. Hier sollte zukünftige Forschung ansetzen und insbesondere mit TrackingVerfahren und angeschlossenen Inhaltsanalysen arbeiten (ggf. ergänzt durch Experience Sampling), um die tatsächlich rezipierten Inhalte und ihre Bedeutung für Rezipient_innen mit Vertrauen in Journalismus in Beziehung zu setzen. Solche Studien wären insbesondere als Langzeit-Untersuchungen durchzuführen, um kausale Effekte nachweisen zu können.

Damit ist auch die vielleicht wichtigste Limitation der vorliegenden Studie angesprochen: aufgrund des Querschnitt-Designs sind kausale Aussagen zu den hier gefundenen Zusammenhängen nicht möglich. Auch wenn an einigen Stellen plausible Vermutungen angestellt werden können und es sich in vielen Fällen vermutlich um Wechselbeziehungen ohne klare Kausalitätsrichtung handelt, sind kausale Nachweise der gefundenen Zusammenhänge dennoch dringend nötig, vor allem im Bereich des Zusammenhangs von Online-Nachrichtennutzung und Vertrauen in Journalismus. Hierfür sollten vor allem verstärkt Panel-Langzeitstudien durchgeführt werden, um die Entwicklung des Vertrauens in Journalismus im Zusammenspiel mit veränderter Informationsnutzung untersuchen zu können. Dabei können auch experimentelle (Langzeit-)Untersuchungen helfen, vor allem um die hier angenommenen Effekte alternativer Ereignisdarstellungen und Medienkritik auf Vertrauen in Journalismus einer Überprüfung zu unterziehen. Dabei sollte Vertrauen in Journalismus allerdings dezidiert als relativ stabiles, langfristiges Konstrukt operationalisiert werden und vor allem getrennt von Qualitätswahrnehmungen erhoben werden. Die Arbeit hat gezeigt, dass es sowohl theoretisch wie auch empirisch äußerst sinnvoll ist, Vertrauen und Qualitätswahrnehmungen zu trennen und Vertrauen nicht als Qualitätswahrnehmung $\mathrm{zu}$ operationalisieren. Einmalige Experimentalstimuli scheinen damit zur Untersuchung von Effekten auf Vertrauen nicht gut geeignet. Vielmehr wäre genauer zu untersuchen, wie Qualitätswahrnehmungen und Vertrauen zusammenspielen und sich gegenseitig beeinflussen. Die vorliegende Studie hat Qualitätswahrnehmungen ebenso wie Personenmerkmale oder Informationsnutzung als Prädiktoren für Vertrauen untersucht. Weitere Forschung könnte hier ansetzen und Qualitätswahrnehmungen als Mediator begreifen und in Langzeitstudien untersuchen. Vermutlich wird z. B. der Kontakt mit alternativen Ereignisdarstellungen und Medienkritik zunächst Qualitätswahrnehmungen beeinflussen, die im Gegenzug Einfluss auf das Vertrauen nehmen. Dieser Weg wurde in der vorliegenden Arbeit aufgrund der Querschnittdaten nicht beschritten.

Weitere Einschränkungen der vorliegenden Studie betreffen vor allem das Sampling. Da das Ziel der qualitativen Vorstudie darin lag, das gesamte Spektrum aus Vertrauen und Misstrauen in Journalismus abzubilden, sind insbesondere die Extremgruppen mit sehr niedrigem und sehr hohem Vertrauen nur von wenigen Personen besetzt. Zusätzlich sind Personen mit niedriger Bildung deutlich unterrepräsentiert. Wenngleich viele relevante Erkenntnisse und Impulse für 
die quantitative Untersuchung gewonnen werden konnten, bleiben damit dennoch einige Personengruppen unberücksichtigt. Ähnliche Einschränkungen gelten auch für das Sampling der quantitativen Stichprobe. Einige Nutzergruppen sind kaum in Online-Panels repräsentiert, vor allem ältere Menschen und tendenziell auch niedriger gebildete. Hier muss zukünftige Forschung ansetzen und auch für schwer zugängliche Bevölkerungssegmente Sampling-Strategien entwickeln.

Insgesamt konnte die vorliegende Studie damit einen Beitrag zum Schließen einiger der identifizierten Forschungslücken in der Forschung zu Vertrauen in Journalismus leisten. Dennoch bleiben einige Forschungsdefizite, die weiterer Forschung bedürfen. Abseits neuer empirischer Erhebungen wäre der Forschung $\mathrm{zu}$ Vertrauen in Journalismus aber zu wünschen, dass die aktuell bestehende Forschung noch stärker systematisiert wird (etwa in Forschungsüberblicken) oder in Meta-Analysen zusammengetragen wird. Mittlerweile liegen deutlich mehr Einzelbefunde zum Vertrauen in journalistische Medien vor, als noch vor einigen Jahren. Woran es noch fehlt, sind integrierte Überblicke über das Feld und eine kritische Zusammenschau dessen, was wir über das Vertrauen in Journalismus wissen und was nicht.

\subsection{Vertrauen erhalten und zurückgewinnen - Schlussbetrachtung und Handlungsempfehlungen}

Eine demokratische Gesellschaft braucht hochwertige Medien, die ihrer Informationsfunktion nachkommen, relevante Themen auf die Agenda setzen und Entwicklungen in Politik, Wirtschaft und Gesellschaft kritisch begleiten. Sie sichern damit die unabhängige Meinungsbildung der Bürger_innen und ermöglichen überhaupt erst eine freiheitlich-demokratische Gesellschaftsordnung. Diese Leistung muss nicht notwendigerweise durch klassische, professionell-journalistische Medien erbracht werden. Neben journalistischen Medien findet sich in digitalisierten Öffentlichkeiten eine Reihe von Alternativen, die teilweise funktional äquivalente oder komplementäre Inhalte anbieten und die Funktion der Selbstbeobachtung der Gesellschaft potenziell umsetzen oder unterstützen können. Zumindest gegenwärtig gibt es jedoch noch keine erkennbaren Strukturen, die die Informationsfunktion des klassischen Journalismus vollständig ersetzen können; dies ist allenfalls in Nischen- und Special-Interest-Bereichen möglich. Für einen breiten, integrierten Nachrichtenüberblick zu aktuellen politischen und gesellschaftlichen Themen sind journalistische Medien damit aktuell unverzichtbar. Nur journalistische Medien haben derzeit die Ressourcen, die Zugänge, die Kompetenzen und die rechtlichen Rahmenbedingungen, um diese Informationsfunktion zu erfüllen. Damit leisten sie einen wichtigen Beitrag zur Integration 
der Gesellschaft, denn nur wenn es eine Instanz gibt, die unabhängig von Partikularinteressen Problemen gesamtgesellschaftliche Relevanz zuschreibt und einen Raum für die Verhandlung ihrer Lösungen bietet, kann die liberale Demokratie existieren. Damit einher geht jedoch auch die große Verantwortung und Verpflichtung des Journalismus, diese Funktionen ernst zu nehmen, journalistische Qualität zu sichern und zu verbessern.

Die aktuelle Konfiguration von Öffentlichkeit ist stark durch weitere Informationsquellen geprägt, die die Deutungshoheit des Journalismus angreifen: Alternativmedien, öffentliche Bürgerkommunikation und Originalquellen werden im Netz noch an Bedeutung zunehmen und gemeinsam mit dem Journalismus ein Informations-Ökosystem bilden, in dem der Journalismus nicht mehr als einziger Gatekeeper auftritt. Damit wandelt sich seine Rolle dramatisch; er muss sich stärker um Einordnung, Orientierung und Überblick bemühen. Im Wettbewerb mit anderen Anbietern wird er notgedrungen transparenter: Fehler, Konstruktionen und systematische Verzerrungen werden häufiger aufgedeckt und die Qualität des Journalismus wird besser überprüfbar. Unter Online-Bedingungen sind journalistische Medien daher herausgefordert, auf die Sicherstellung ihrer Qualität zu achten und aktiv am Erhalt und Aufbau von Vertrauen zu arbeiten. Vertrauen verleiht ihnen die gesellschaftliche Legitimation, ermöglicht eine ökonomische Grundlage und ist damit Kernvoraussetzung für ihre Existenz. Es gilt damit für den Journalismus in den kommenden Jahren, Vertrauen aufrechtzuerhalten und in bestimmten Milieus auch zurückzugewinnen. Aktuell zehren viele journalistische Anbieter noch von einem Vertrauensvorschuss aus prädigitalen Zeiten, verlassen sich auf den Nimbus ihrer Marken und stellen sich zu wenig auf die gewandelte Informationsumgebung ein. Welche konkreten Handlungsempfehlungen lassen sich daher aus den empirischen Ergebnissen dieser Arbeit ableiten, um das Vertrauen in den Journalismus aufrecht zu erhalten bzw. zurückgewinnen zu können?

\section{Handlungsfeld 1: Realitätskonstruktion hinterfragen und Orientierung bieten}

Beim Publikum ist die Erkenntnis bereits stark verankert, dass der Journalismus nicht mehr die einzige Instanz zur Herstellung von Öffentlichkeit ist. Das gilt vor allem für das Milieu gebildeter, politisch interessierter und gut informierter Medienskeptiker (siehe Kap. 7.2). Der Journalismus scheint sich mit diesem Wandel noch nicht überall arrangiert zu haben. Zum Erhalt und Aufbau von Vertrauen muss es daher gelten, alternative Ereignisdarstellungen als Teil der neuen Informations-Ökologie zu akzeptieren, wo sinnvoll auf ihren Wahrheitsgehalt zu überprüfen und als Beiträge zum öffentlichen Diskurs ernst zu nehmen. Damit ist natürlich nicht gemeint, Desinformation, Unsinn und Gerüchten eine Bühne zu geben, sondern offen für alternative Weltdeutungen zu sein. Das wird auch bedeuten, die eigenen kognitiven und sozialisatorischen Verzerrungen und Mecha- 
nismen der Konstruktion von Realität zu hinterfragen und wo nötig anzupassen. Ein konkretes Beispiel für einen konstruktiven Umgang mit diesem Wandel sind etwa Artikel im Stile von ,was wir wissen und was nicht', in denen zu aktuellen Ereignissen die derzeit gesicherten Kenntnisse ebenso wie kursierende Falschmeldungen oder Verschwörungstheorien aufgegriffen und einem Faktencheck unterworfen werden (siehe etwa Reinbold, Reuter, \& Sydow, 2017 zum Thema Gasangriff in Syrien). In diese Richtung weisen auch Fact-Checking-Einheiten wie der ARD-Faktenfinder, der explizit alternative Darstellungen aufgreift, recherchiert und damit dem Publikum Orientierung in der Vielfalt der Ereignisdarstellungen bietet.

Wie die vorliegende Arbeit gezeigt hat, beginnt die bewusste Suche nach Alternativen zu journalistischen Darstellungen bei Unzufriedenheit mit der Berichterstattung oder einem wahrgenommenen Informationsdefizit, was zu einer Spirale des Misstrauens führen kann (siehe Kap. 7.5). Solche Leerstellen kommen zustande, wenn journalistische Medien bestimmte Themen nicht aufgreifen oder keine zentrale Anlaufstelle für Informationen bieten, so dass verschwörungstheoretische, staatliche oder sonstige interessensgeleitete Akteure die einzigen Anbieter von Informationen zu einem bestimmten Thema bzw. Themenaspekt sind. Golebiewski und Boyd (2018) sprechen hier von „,data voids“, die im Internet entstehen. Diese Leerstellen gilt es für den Journalismus zu besetzen und so Zweifel vorwegzunehmen. So könnten Online-Medien z. B. spezielle InfoSeiten oder Wikis zu aktuellen Themen anbieten, wo einerseits eigene Inhalte gesammelt und andererseits weiterführende Links zu seriösen Quellen angeboten werden. Durch Suchmaschinen- und Social Media-Optimierung sollten diese Inhalte insbesondere den Personen zugänglich gemacht werden, die nicht direkt auf die Inhalte journalistischer Medien zugreifen, sondern über unterschiedliche personalisierte Kanäle damit Kontakt haben. So kann sich der Journalismus online stärker als Orientierungsanker zu bestimmten Themen positionieren und aktiv dagegen arbeiten, Nutzer_innen an nicht-journalistische Anbieter zu verlieren. Davon dürften auch die Reichweiten journalistischer Medien profitieren, zumal nach Daten des Reuters Digital News Reports in Deutschland 40 Prozent der Onliner angeben, im Internet hauptsächlich über Suchmaschinen auf Nachrichten zu stoßen (Nielsen \& Schrøder, 2014, S. 483).

\section{Handlungsfeld 2: Transparenz, Aufklärung und Bildung}

Um als ein solcher Orientierungsanker fungieren zu können, wird der Journalismus auch stärker auf Transparenz setzen müssen. Unter Online-Bedingungen wird die journalistische Realitätskonstruktion durchschaubarer, angreifbarer und muss sich mit Alternativen messen. Daher gilt es im Journalismus mehr denn je, zu erklären und nachvollziehbar zu machen, wie journalistische Ereignisdarstellungen zustande kommen. Misstrauen in Journalismus wird am stärksten von der 
Wahrnehmung geprägt, dass journalistische Fehler absichtlich zustande kommen und auf Manipulationen zurückzuführen sind (siehe Kap. 7.4.2 und 9.3). Hier muss noch besser daran gearbeitet werden, dass solche Vorwürfe keine Berechtigung haben. Der Fall manipulierter Geschichten durch den Spiegel-Reporter Claas Relotius verweist exemplarisch auf notwendige Veränderungen im Journalismus. So stellte die Aufklärungskommission beim Spiegel fest, dass es im Haus an Transparenz, kritischer Prüfung und Dokumentation sowie einer Fehlerkultur mangele, die diesen Betrug erst ermöglichten (Fehrle, Höges, \& Weigel, 2019). Diese Probleme sind sicher nicht auf den Spiegel beschränkt, so dass zu hoffen ist, dass der Fall die gesamte Branche zu Verbesserungen anregt. Andererseits muss auch versucht werden, haltlosen Vorwürfen der Abhängigkeit und Manipulation den Wind aus den Segeln zu nehmen. Hier muss der Journalismus vor allem deutlich bessere Erklärungs- und Aufklärungsarbeit leisten. Dass nur 40 Prozent der Befragten der vorliegenden Studie wissen, dass ein Bericht über einen Bundesminister nicht von ihm zur Veröffentlichung freigegeben werden muss, ist ein Armutszeugnis weniger für die Bürger_innen, sondern in erster Linie für den Journalismus und seine Leistungen bei der Erklärung der eigenen Arbeit (siehe Kap. 9.2.1). Dabei zeigte sich in der vorliegenden Studie, dass Aufklärung über journalistische Arbeit auch bei skeptischen Menschen auf fruchtbaren Boden fallen kann: Viele von ihnen haben großes Interesse an aktuellen politischen und gesellschaftlichen Ereignissen, informieren sich ausführlich auch in journalistischen Medien und sind dabei auch an den Mechanismen der Nachrichtenproduktion interessiert. Initiativen wie Transparenz-Blogs, in denen Redaktionen über ihre Arbeit berichten, wie etwa beim Spiegel (Hans, 2017) oder der ZEIT (Wegner, 2016), sind also durchaus sinnvolle Werkzeuge, um zumindest einen Teil der medienskeptischen Personen zu erreichen. Sie müssen aber noch ausgebaut und besser in die tägliche Berichterstattung integriert werden, um auch bei weniger interessierten Personen anzukommen. So wurde etwa erfolgreich mit kurzen Erklär-Boxen experimentiert, die neben einem Nachrichtenartikel Informationen zur Motivation für eine Recherche und deren Hintergründe darlegen. Sie konnten in einer experimentellen Befragung die wahrgenommene Vertrauenswürdigkeit des Mediums steigern (Chen, Curry, \& Whipple, 2019). Darüber hinaus liegt es auch in der Verantwortung der etablierten journalistischen Medien selbst, den Medienjournalismus zu stärken und einen kritischen, aber auch erklärenden Blick auf die eigene Branche zu werfen, um Rezipient_innen auch hier Orientierung zu ermöglichen und Hintergrundwissen bereitzustellen. Nicht zuletzt ist die Medienkompetenzbildung in Schulen gefordert, stärker den Entstehungsprozess von Nachrichten zu erklären und hier Bewusstsein für die Probleme und Uneindeutigkeiten journalistischer Arbeit zu schaffen. Damit sind explizit nicht nur Angebote für Schüler_innen, sondern gerade auch notwendige (Weiter-)Bildungsangebote für Lehrer_innnen gefordert. 
Medienkompetenzbildung darf allerdings nicht nur an den Schulen angesiedelt sein und sich auf jüngere Menschen richten, sondern muss auch ältere Personen ansprechen. Gerade online fallen älteren Menschen ohne digitale Sozialisation Glaubwürdigkeits- und Vertrauensurteile zu Nachrichten schwer, was sich etwa darin zeigt, dass Personen über 65 Jahren überwiegend für die Verbreitung von ,Fake News' verantwortlich sind (Guess et al., 2019, S. 3).

\section{Handlungsfeld 3: Repräsentation und Vielfalt}

Als wichtiger Einflussfaktor auf Vertrauen in Journalismus hat sich außerdem herausgestellt, inwiefern Menschen ihre eigene Meinung in den Medien repräsentiert sehen. Dieses mediale Repräsentationsgefühl ist insbesondere für das instrumentelle Vertrauen bedeutsam, also die Bereitschaft, sich eine Meinung aufgrund journalistischer Medien zu bilden und Entscheidungen basierend auf ihren Informationen $\mathrm{zu}$ treffen. Dies sind die demokratietheoretisch besonders bedeutsamen Aspekte des Vertrauens in Journalismus, so dass ein Verlust instrumentellen Vertrauens besonders dramatisch ist. Mangelndes mediales Repräsentationsgefühl ist zwar in allen Bevölkerungsschichten verbreitet, findet sich aber besonders stark im AfD-Milieu. Damit einhergehend lautet der zentrale Vorwurf vieler Menschen an die Medien, der Meinungskorridor sei zu eng und es würde in den Medien ein bürgerlich-linksliberales Gesellschaftsverständnis vorherrschen. Medienkritische Einstellungen sind damit auch Ausdruck einer generellen Unzufriedenheit mit dem demokratischen System, als dessen Repräsentant viele Bürger_innen auch den Journalismus sehen (siehe Kap. 7.1). Hinter diesen Einstellungen stehen offenbar tiefgreifende Erfahrungen mangelnder gesellschaftlicher Repräsentation, Teilhabe und Wertschätzung, was Patzelt (2015) als „Repräsentationslücke“ bezeichnet. Dieser Befund sollte auch dem Journalismus zu denken geben. Es ist nicht von der Hand zu weisen, dass im durchschnittlichen Mainstream-Journalismus in Themenauswahl, Berichterstattung und Kommentierung eher weltoffene, globalisierungs- und liberalisierungsfreundliche Positionen vorherrschen (Müller, 2018, S. 5). Da sich die meisten Journalist_innen aus hoch gebildeten, bürgerlich-liberalen Milieus rekrutieren, ist dieser Befund auch naheliegend. Wenn sich dadurch allerdings breite Bevölkerungsgruppen in den etablierten Medien nicht mehr repräsentiert sehen und Anschluss bei Alternativmedien und anderen Informationsquellen im Internet suchen, hat das eine potenziell desintegrierende Wirkung. Die Konsequenz kann nun selbstverständlich nicht sein, Rechtspopulist_innen und den Feinden einer offenen Gesellschaft das Wort zu reden und ihre Argumente zu reproduzieren. Der Journalismus muss vielmehr die gesellschaftlichen und politischen Probleme ernst nehmen, die hinter den Wahrnehmungen mangelnder Repräsentation und Teilhabe stehen (Müller, 2018, S. 8). Dabei wird es gelten, ohne Paternalismus und Abwertung ein Verständnis für die Lebenswelten der Menschen zu entwi- 
ckeln, die solchen Einstellungen anhängen - und diese auch stärker medial zu repräsentieren. Insofern ist im Journalismus einerseits inhaltlich auf Vielfalt zu setzen, andererseits auch die bestehende Vielfalt stärker zu betonen und prominenter zu platzieren. Dies gilt auch personell: Noch immer sind Redaktionen relativ homogen aus der Mittelschicht besetzt, Personen aus Arbeiter_innenmilieus oder mit Migrationshintergrund sind hingegen deutlich unterrepräsentiert (Weischenberg et al., 2006, S. 69; Borchart, Lück, Kieslich, Schultz, \& Simon, 2019, S. 36-47). Hier wird es in Zukunft gelten, mehr Personen mit vielfältigen Sozialisationserfahrungen für den Beruf Journalismus zu gewinnen, um die mediale Repräsentationslücke schließen zu können.

Das Vertrauensverhältnis von Journalismus und Publikum ist jedoch nicht ausschließlich Bringschuld des Journalismus. Journalistische Medien haben zwar die vornehmliche Verantwortung, für das Publikum vertrauenswürdige Arbeit zu leisten. Im Sinne der Befragten der qualitativen Teilstudie kann aber auch die Eigenverantwortung des Publikums eingefordert werden. Auch die Bürger_innen sind aufgefordert, sich mit dem Journalismus auseinanderzusetzen, seine wichtige Rolle anzuerkennen und eine konstruktive, aber kritische Haltung zu entwickeln. Nur mit gegenseitiger Wertschätzung von Journalismus und Zivilgesellschaft bei gleichzeitig kritischer Beobachtung ist es möglich, die wichtigen Funktionen des Journalismus für liberale Demokratien auch unter gewandelten Bedingungen von Öffentlichkeit zu erhalten und zu stärken.

Open Access Dieses Kapitel wird unter der Creative Commons Namensnennung 4.0 International Lizenz (http://creativecommons.org/licenses/by/4.0/deed.de) veröffentlicht, welche die Nutzung, Vervielfältigung, Bearbeitung, Verbreitung und Wiedergabe in jeglichem Medium und Format erlaubt, sofern Sie den/die ursprünglichen Autor(en) und die Quelle ordnungsgemäß nennen, einen Link zur Creative Commons Lizenz beifügen und angeben, ob Änderungen vorgenommen wurden.

Die in diesem Kapitel enthaltenen Bilder und sonstiges Drittmaterial unterliegen ebenfalls der genannten Creative Commons Lizenz, sofern sich aus der Abbildungslegende nichts anderes ergibt. Sofern das betreffende Material nicht unter der genannten Creative Commons Lizenz steht und die betreffende Handlung nicht nach gesetzlichen Vorschriften erlaubt ist, ist für die oben aufgeführten Weiterverwendungen des Materials die Einwilligung des jeweiligen Rechteinhabers einzuholen.

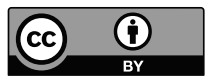

